\title{
Cardiac CT Angiography in Congestive Heart Failure
}

\author{
Avi Levine and Harvey S. Hecht \\ Icahn School of Medicine, Mount Sinai Medical Center, New York, New York
}

Cardiac CT angiography has become an important tool for the diagnosis and treatment of congestive heart failure. Differentiation of ischemic from nonischemic cardiomyopathy; evaluation of myocardial perfusion; characterization of hypertrophic cardiomyopathy, left ventricular noncompaction, and arrhythmogenic right ventricular dysplasia; and delineation of congenital heart defects and valvular abnormalities are the primary diagnostic applications. Therapeutic use includes visualization of the coronary venous anatomy for optimal implementation of cardiac resynchronization therapy and evaluation of left ventricular assist devices and transplant vasculopathy.

Key Words: cardiology; image reconstruction; congestive heart failure; cardiac CTA; noninvasive imaging

J Nucl Med 2015; 56:46S-51S

DOI: 10.2967/jnumed.114.150441

H any structural or functional impairment of ventricular filling or ejection of blood (1). It affects approximately 5.1 million people in the United States, with greater than 650,000 cases diagnosed annually, and those numbers are expected to rise in the future (2). Heart failure is a progressive disease that leads to significant morbidity and mortality and accounts for more than 1 million hospitalizations annually, with a 5-y mortality of approximately $50 \%$ (3). Multidetector cardiac CT angiography (CTA) is an ever-advancing technology, having evolved from the early 4-slice acquisition systems to the modern multislice cardiac CT. With up to 320 simultaneous slices and faster gantry rotational systems, cardiac CT allows for improved temporal and spatial resolution. Entire examinations can be completed in less than $5 \mathrm{~s}$, and the volume of contrast material required for comprehensive examinations continues to decrease. This review summarizes the many potential applications of CTA for patients with heart failure.

\section{DIAGNOSIS OF HEART FAILURE}

\section{Reduced Ejection Fraction (EF)}

The identification of reduced left ventricular (LV) function is at the core of the diagnosis of heart failure with reduced EF. Reduced LV function can be detected by multiple modalities, including transthoracic echocardiography, the modality most commonly

Received Jan. 6, 2015; revision accepted Mar. 27, 2015.

For correspondence or reprints contact: Harvey S. Hecht, Icahn School of Medicine, Mount Sinai Medical Center, One Gustave L. Levy PI., Box 1030, New York, NY 10029-6574.

E-mail: harvey.hecht@mountsinai.org

COPYRIGHT (c) 2015 by the Society of Nuclear Medicine and Molecular Imaging, Inc. used for diagnosis; transesophageal echocardiography; cardiac MR imaging; left ventriculography during cardiac catheterization; radionuclide ventriculography; and SPECT.

CTA can also be used to diagnose reduced LV function. With retrospective imaging techniques, continuous multidetector image acquisition with electrocardiogram gating allows for the reconstruction of images at multiple instants in the cardiac cycle. With shortaxis reconstruction at every $5 \%$ or $10 \%$ of the R-R interval, both LV and right ventricular (RV) volumes can be calculated, allowing for determination of the EFs of both ventricles (Fig. 1). EF calculation by CTA correlates well with echocardiographic assessment of LV function (4). Multiple studies have also shown a strong correlation between CTA and cardiac MR imaging for EF calculation (5-7). However, CTA has a more limited temporal resolution than cardiac MR imaging, resulting in slight overestimations of end-systolic volume and EF, especially in patients with a reduced EF (7). Nonetheless, according to American College of Cardiology Foundation/ American Heart Association guidelines for the diagnosis and management of heart failure, CTA may be useful for assessing cardiac function and wall motion (1).

\section{Regional Wall Motion Abnormalities}

Aside from measuring overall contractility, CTA is also useful for the diagnosis of regional wall motion abnormalities. With a cine-loop format to display images, regional wall motion can be evaluated. Several studies have confirmed the usefulness of CTA for evaluating LV regional wall function $(4,8-10)$. CTA has a high specificity for detecting wall motion abnormalities, and it appears to have the highest accuracy for detecting wall motion abnormalities in the left anterior descending and left circumflex arteries (11). Its accuracy for the identification of regional wall motion abnormalities approaches the accuracy of cardiac MR imaging (12).

The ability of CTA to measure ventricular volumes and EF is not limited to the left ventricle. CTA can be useful for evaluation of the right ventricle, which can be technically difficult to evaluate by other imaging modalities, especially echocardiography. RV volumes and EF calculations have been shown to correlate well with those obtained from cardiac MR imaging, with a correlation coefficient of greater than 0.96 (13). Therefore, for patients with limited echocardiographic windows or for patients who cannot undergo cardiac MR imaging because of device implantation, CTA is currently recommended for the evaluation of RV morphology and function (14).

\section{Heart Failure with Preserved EF}

Heart failure encompasses more than just reduced LV function. It has been estimated that approximately $50 \%$ of patients with clinical heart failure have normal systolic function (1). This disease entity, termed heart failure with preserved EF, is the result of abnormalities in myocardial diastolic properties. Multiple echocardiographic indices, including mitral valve flow velocities and tissue Doppler velocities, are used for the diagnosis of heart failure with 


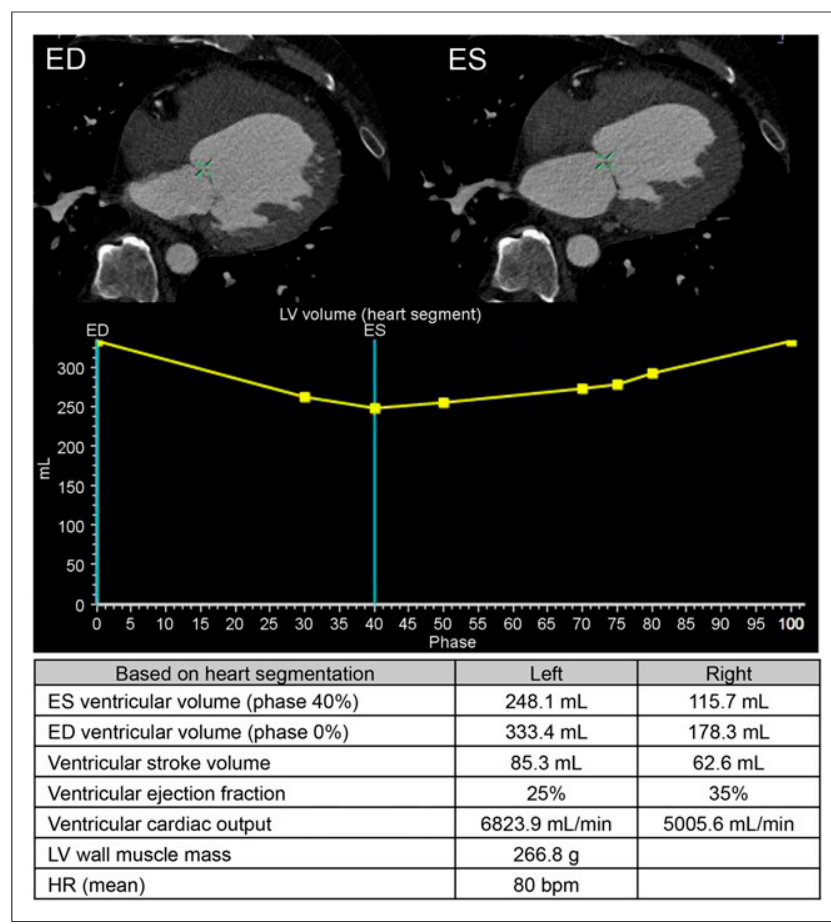

FIGURE 1. Nonischemic cardiomyopathy in 65-y-old man who had new-onset dyspnea on exertion and atypical chest pain. Cardiac CTA revealed no significant obstructive disease and ejection fraction of $25 \%$. Cardiac catheterization was not performed. (Top) End-diastolic (ED) and end-systolic (ES) frames. (Middle) LV volume curve. (Bottom) Derived CAT measurements. $\mathrm{HR}=$ heart rate.

preserved EF. However, it may be feasible to use CTA for measurement of the diastolic properties of the heart. In an elegant study, Boogers et al. (15) used mathematic analyses to calculate transmitral valve flow velocities and mitral valve ring motion velocities at the septum. These measurements showed a good correlation with echocardiographically derived measures of diastolic function, with an overall accuracy of $79 \%$. Although not currently used for this disease process, CTA may play a role in the diagnosis of heart failure with preserved EF in the future, especially for patients already undergoing CTA for coronary artery evaluation.

\section{ETIOLOGY OF HEART FAILURE}

Once the diagnosis of heart failure has been established, it is important to establish the etiology of the disease. There are many possible underlying causes for heart failure, both cardiac and noncardiac. Identification of the condition responsible may be important because some conditions that lead to LV dysfunction are potentially treatable or reversible.

\section{Ischemic Versus Nonischemic Cardiomyopathy}

One of the most important initial diagnostic steps in the workup of heart failure is the distinction between ischemic cardiomyopathy and nonischemic cardiomyopathy. The belief that coronary artery disease (CAD) is the underlying cause in approximately two-thirds of patients with heart failure (16) has important therapeutic and prognostic implications, as revascularization can favorably affect LV function in some patients with impaired yet viable myocardium (17).

It is difficult to clinically distinguish between ischemic cardiomyopathy and nonischemic cardiomyopathy without further diagnostic workup. Patients with nonischemic cardiomyopathy may have symptoms of chest pain or electrocardiogram changes consistent with CAD. In contrast, patients with ischemic cardiomyopathy may have heart failure and no symptoms of chest pain. In addition, specific echocardiographic findings may not always be reliable in helping to establish a diagnosis. Segmental wall motion abnormalities are common in dilated cardiomyopathy, even in the absence of obstructive CAD (Fig. 1) (18).

Invasive coronary angiography (ICA) is considered the gold standard for the diagnosis of ischemic cardiomyopathy. In patients with newly diagnosed heart failure and anginal symptoms, coronary angiography is recommended (1). However, ICA is an invasive study that carries a risk for complications, most importantly, vascular access site complications, with bleeding rates ranging from $0.05 \%$ to $2.3 \%$, as well as death, myocardial infarction, and stroke, with rates approaching $3 \%$ (19). Therefore, noninvasive modalities are a reasonable initial diagnostic step (1).

Assessment of coronary artery calcium (CAC), without the need for intravenous contrast material administration, can provide important diagnostic data. Multiple studies have shown the value of CAC scores in excluding CAD as the etiology for heart failure (20-22). In patients with a diagnosis of heart failure, an Agatston score of 0 has been shown to have $100 \%$ specificity in excluding "high-risk CAD," defined as left main coronary artery stenosis or stenosis in at least 2 major epicardial coronary arteries $(20,22)$. Although the clinical utility of CAC scores in patients with heart failure has not been well described, assessment of CAC should be considered as an initial study to exclude an ischemic etiology for heart failure.

Beyond providing an assessment of CAC, CTA can provide a direct noninvasive evaluation of the coronary arteries with intravenous contrast material. Compared with invasive angiography, CTA had high qualitative and quantitative accuracy in the general population (23). CTA has also been shown to be feasible, safe, and accurate for detecting CAD with high sensitivity and specificity in patients with dilated cardiomyopathy (24). A metaanalysis by Bhatti et al. (25) demonstrated that CTA is a highly accurate diagnostic modality for excluding an ischemic etiology in patients with cardiomyopathy of undetermined cause, with a sensitivity of $98 \%$ and a specificity of $97 \%$. In fact, CTA has been accepted as an alternative to ICA for evaluating coronary arteries in patients with new-onset heart failure to assess etiology (25). In an ongoing randomized clinical trial, IMAGE HF Project 1-C, CTA and ICA are being compared for patients with heart failure requiring coronary anatomic definition, endpoints of diagnostic accuracy, clinical outcomes, and resource use (26).

The use of CTA for the assessment of coronary arteries is not limited to patients with normal sinus rhythm. Atrial fibrillation is a common comorbid condition in patients with heart failure, with a prevalence approaching $50 \%$ in patients with more advanced heart failure (27). Patients with atrial fibrillation have been excluded from most CTA studies because the test requires a low heart rate, and the beat-to-beat variations in atrial fibrillation can lead to poor image quality and make image reconstruction difficult. Nonetheless, a metaanalysis by Vorre and Abdulla (28) showed that CTA has high diagnostic accuracy in patients with atrial fibrillation. However, patients with atrial fibrillation did require a higher effective radiation dose than patients with normal sinus rhythm.

CTA can also provide more than just an assessment of the coronary arteries in patients with ischemic cardiomyopathy. With first-pass injection of contrast material, CTA imaging has the ability 
to determine resting myocardial perfusion defects. Compared with myocardial perfusion imaging, CTA accurately detected perfusion defects (29). Compared with ICA, CTA had a low positive predictive value, likely the result of image artifacts; however, it had a notable ability to exclude flow-limiting lesions, with a negative predictive value of greater than $85 \%$ (30).

Beyond the differentiation between ischemic cardiomyopathy and nonischemic cardiomyopathy, other cardiomyopathy subtypes have distinctive changes that can be detected by CTA. In infiltrative cardiomyopathies, such as amyloidosis or sarcoidosis, subtly heterogeneous attenuation of the myocardium can be seen on CTA. In hypertrophic cardiomyopathy, CTA can show increased wall thickness and can delineate the exact location and extent of myocardial involvement. LV noncompaction can also be identified by CTA, with prominent trabeculations and areas of noncompaction (Fig. 2). In addition, in arrhythmogenic RV dysplasia, CTA can demonstrate fat in the RV wall and abnormal RV wall motion (Fig. 3) (31).

CTA can also be useful for patients with congenital heart disease. More patients with congenital heart defects are living into adulthood and, not uncommonly, can develop heart failure as a consequence of their underlying cardiac abnormalities. CTA can provide a highquality assessment of congenital anatomy, prior surgical repair, and progression of underlying congenital conditions as well as structural and anatomic details of coronary artery anomalies $(32,33)$.

\section{Valvular Heart Disease}

Valvular heart disease is a common cause of heart failure. A full description of the use of CTA for the diagnosis and management of valvular heart disease is beyond the scope of this review, but a brief discussion of the use of CTA for left-side valvular lesions, the most common cause of valve-related heart failure, is warranted.

CTA can be useful for the assessment of aortic valve lesions. In aortic regurgitation, the aortic regurgitant orifice area can be calculated by planimetry with CTA, and quantification of the regurgitant orifice area has been shown to correlate well with echocardiography in determining the severity of aortic regurgitation (34). The severity of aortic stenosis can also be evaluated effectively by use of CTA for planimetry of the aortic valve area during the

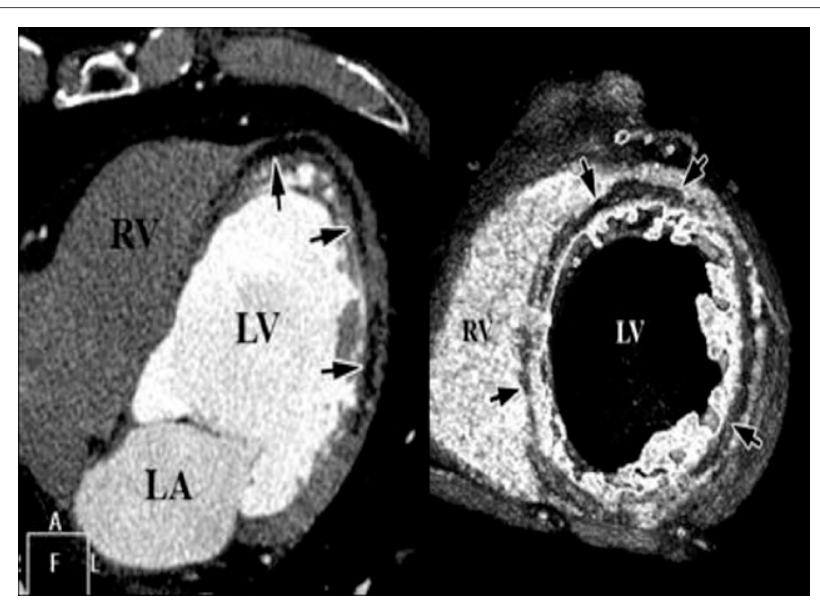

FIGURE 2. LV noncompaction. (Left) Axial image of heart shows deep intertrabecular recesses (arrows) at apex and lateral wall of left ventricle. (Right) Short-axis volume-rendered image of heart shows areas of low Hounsfield units within LV wall (arrows), which are likely to be fibroelastic infiltrations of noncompaction. $L A=$ left atrium; RV = right ventricle.

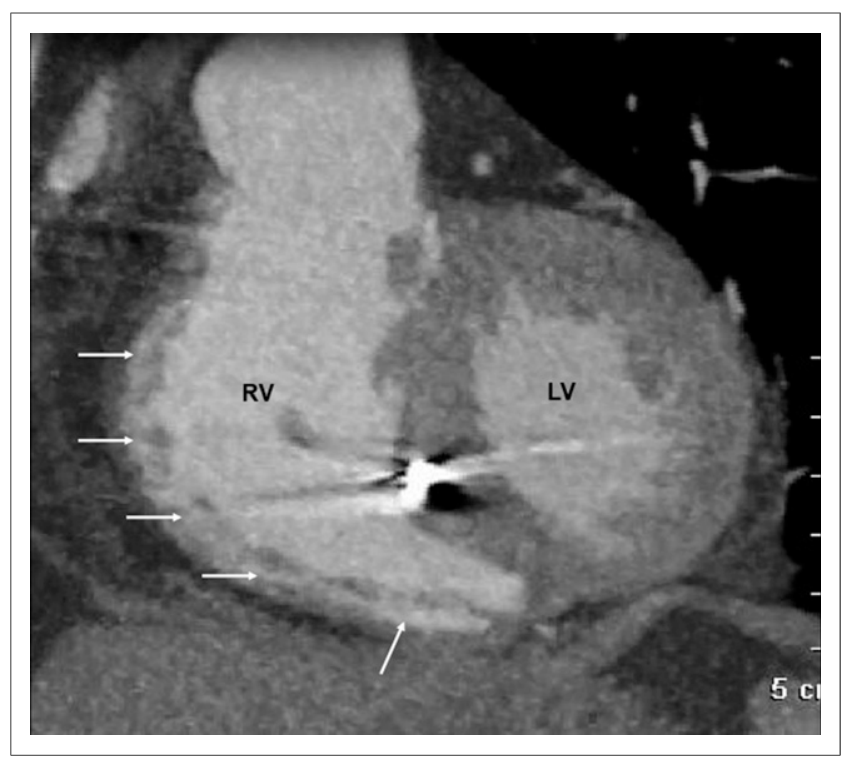

FIGURE 3. Arrhythmogenic RV dysplasia. Arrows highlight areas of fat in dilated right ventricle (RV). $L V=$ left ventricle.

systolic phase. Compared with transesophageal echocardiography, CTA provided an accurate measurement of the aortic valve area (35). Beyond diagnosis, CTA can provide critical treatment-related information for aortic valve disease. A comprehensive CTA examination is part of the standard preprocedural protocol at many institutions when transcatheter aortic valve replacement is being considered.

Mitral valve lesions can also be evaluated by CTA. In mitral stenosis, CTA shows thickened leaflets, and planimetry can be used to measure the mitral valve area. Studies have shown that although the mitral valve area measured by CTA planimetry is systematically larger than that calculated by echocardiography, CTA may allow reliable discrimination of mitral valve stenosis severity grades (36). In mitral regurgitation, CTA can show a lack of apposition of the mitral valve cusps at the end of systole, and it allows for direct planimetry of the regurgitant orifice area. CTA also allows for the detection of structural abnormalities of the mitral valve, such as prolapse, flail leaflet, annular calcification, and leaflet thickening. Moreover, in functional mitral regurgitation, CTA can provide anatomic and geometric details of the entire mitral valve apparatus, yielding both diagnostic and therapeutic information.

\section{TREATMENT OF HEART FAILURE}

Beyond the etiology of heart failure, CTA is extremely valuable for various heart failure treatment modalities, including cardiac resynchronization therapy (CRT), left ventricular assist devices (LVADs), and heart transplants.

\section{CRT}

CRT via the implantation of a biventricular pacemaker is a therapeutic option in symptomatic patients with LV dysfunction and a wide QRS complex. This therapy has been shown to improve symptoms and ventricular size and function while decreasing hospitalizations and mortality (37-39). However, a significant percentage of patients receiving CRT therapy do not show any symptomatic benefit and are termed "CRT nonresponders." Among the critical issues in determining the success of CRT therapy are LV dyssynchrony and cardiac venous anatomy. 
CRT therapy involves the recoordination of regional wall contraction, and the presence of LV dyssynchrony at baseline appears to be an important predictor of the response to CRT (40). Echocardiography is the conventional method for the evaluation of LV mechanical dyssynchrony. However, CTA is also able to provide an assessment of mechanical dyssynchrony and was shown to be accurate in a comparison with echocardiography (41). CTA has been proven to be helpful in guiding CRT therapy on this basis (42).

The implantation of a CRT device involves the insertion of an LV pacing lead through the coronary sinus, and the main factor determining the success of LV lead implantation is cardiac venous anatomy (43). A careful assessment of venous anatomy ensures that the latest area of ventricular activation can be reached with the pacemaker lead. The technique most commonly used to evaluate the coronary venous system is retrograde venography by direct manual contrast material injection, but several studies have shown the feasibility of CTA for the noninvasive assessment of cardiac venous anatomy (Fig. 4) $(42,44,45)$. In particular, in patients with abnormal venous anatomy, CTA can provide valuable information on the course of the coronary sinus and its tributaries. CTA can also identify patients in whom surgical epicardial lead placement may be more appropriate from the outset. Moreover, as demonstrated in an elegant study by Wong et al. (46), CTA can also be used for postimplant assessment of lead placement, especially in relation to areas of myocardial scarring, which has been associated with a nonresponse to CRT therapy.

\section{LVAD Assessment}

Because of the growing burdens of heart failure and donor organ shortage and a rise in the number of elderly patients ineligible for heart transplants, LVADs have emerged as an increasingly viable therapy for advanced heart failure (47). They provide a significant mortality benefit over conventional medical therapy; more than 2,000 LVADs were implanted in the year 2013 alone (48). However, with the significant increase in the use of LVADs comes the potential for an increased incidence of associated complications, and detection and evaluation of these complications have important therapeutic implications.

Although other imaging modalities are unable to provide a comprehensive assessment of all LVAD components, CTA provides noninvasive high-resolution imaging of LVADs in

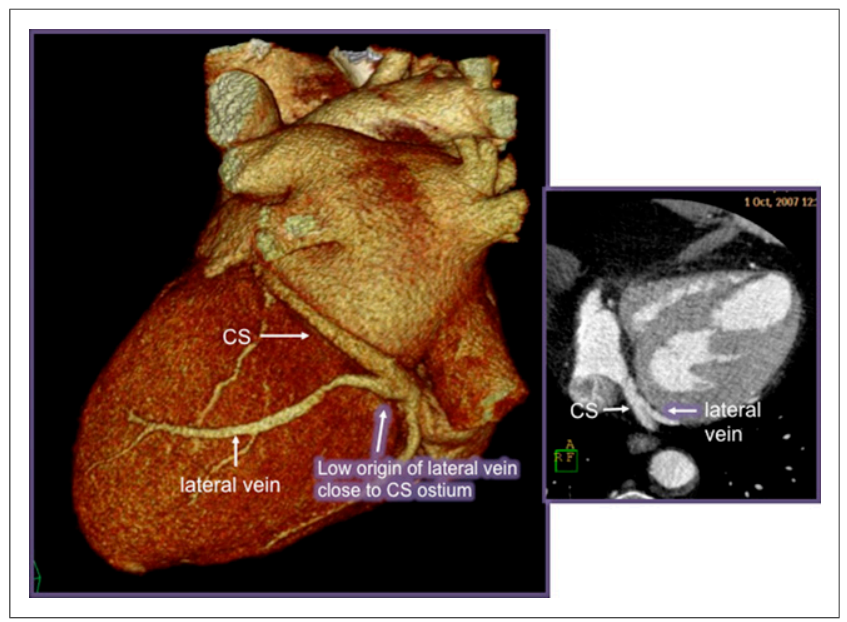

FIGURE 4. Low origin of lateral vein close to coronary sinus (CS) ostium. (Left) Volume-rendered image. (Right) Axial image. their entirety (Fig. 5). The volume of coverage provided by CTA enables direct visualization of inflow and outflow cannulas in their entirety and allows for the evaluation of LVAD placement as well as the recognition of cannula thrombus or deformation. Although some degree of imaging artifacts can be generated by an LVAD, CTA can be used to diagnose LVAD inflow obstruction; multiple studies have proven CTA to be feasible and accurate for detecting inflow and outflow cannula thrombosis and malposition $(49,50)$.

Another application of CTA in patients with LVADs involves assessment of the right ventricle. After LVAD implantation, right heart failure confers significant morbidity and mortality (51). An accurate echocardiographic assessment of RV size and function after LVAD implantation can be technically difficult because of postoperative changes and artifacts from the LVAD itself. CTA, without the limitations of an acoustic window, is highly effective and reproducible for the assessment of RV volume and function in patients with LVADs (52).

CTA may also be able to provide more than just structural data for LVADs. Dynamic CTA with mathematic analyses of flow can be used to determine cardiac output in patients with a continuousflow LVAD. In one study, CTA measurements of cardiac output in the ascending aorta distal to the anastomosis of the outflow cannula correlated with cardiac output measurements by SwanGanz thermodilution during right heart catheterization (53). Although cardiac output calculation by CTA is a potentially exciting future application of CTA for patients with LVAD, the clinical utility of this modality remains to be determined.

\section{Heart Transplant Vasculopathy}

Heart transplantation remains the paramount treatment modality for end-stage heart failure, with a 5-y survival of approximately $70 \%$ (54). However, after heart transplantation, one of the most feared complications is cardiac allograft vasculopathy (CAV), consisting of concentric and diffuse coronary intimal hyperplasia. CAV affects up to $50 \%$ of transplant recipients within $10 \mathrm{y}$, and those with severe CAV have a poor prognosis (55). Therefore,

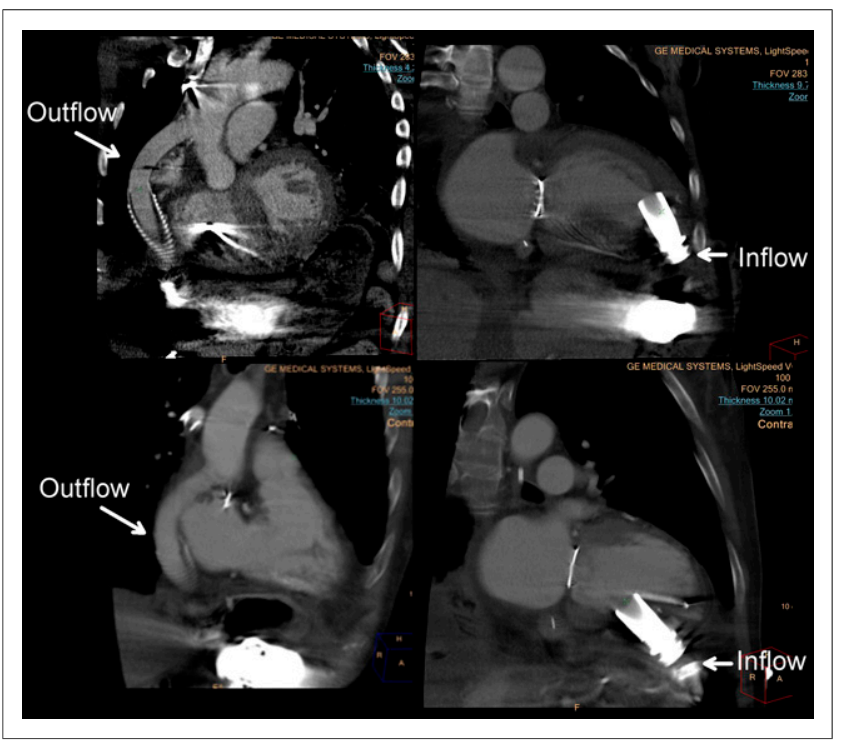

FIGURE 5. LVADs. (Top) Outflow and inflow with typical apical inflow location. (Bottom) Outflow and inflow with atypical interior inflow location. 
routine screening for CAV after transplantation is necessary, especially given its absent or atypical symptomatology.

The diagnosis of CAV has traditionally relied on the use of coronary angiography. Intravascular ultrasound during ICA is the most sensitive method for detecting CAV because it can measure intimal thickening within the coronary artery wall itself (56). Many noninvasive testing modalities, including exercise electrocardiography, stress echocardiography, and myocardial perfusion imaging, are limited by modest diagnostic accuracy and are not recommended as screening tools for CAV (57). However, CTA is safe and feasible for monitoring heart transplant recipients (58), and it provides the theoretic advantage over traditional ICA of being able to visualize both the lumen and the vessel wall simultaneously. A recent metaanalysis (59) demonstrated that modern CTA is a reliable noninvasive imaging alternative for the detection of CAV, even in comparison with intravascular ultrasound, with excellent sensitivity, specificity, and negative predictive value.

\section{LIMITATIONS}

Despite the many advantages of CTA for patients with heart failure, it is important to delineate the limitations of and risks associated with CTA. First and foremost, cardiac CT requires the use of intravenous iodinated contrast material. Beyond the small incidence of an anaphylactic reaction, there is the known risk of nephrotoxicity, which is especially important for patients with heart failure because many patients with end-stage disease have concomitant renal impairment. Second, patients are required to hold their breath for up to $10 \mathrm{~s}$ during image acquisition for CTA. Although this requirement is almost always feasible, it can pose a problem for a patient with decompensated heart failure because such a patient may not be able to perform an adequate breath hold. Another issue is the need for low and regular heart rates, which can directly affect the quality of the study. Patients with heart failure often have frequent premature ventricular contractions, and, as discussed earlier, atrial fibrillation is a common comorbidity in such patients. Finally, CTA involves radiation exposure. The effective radiation dose of CTA has progressively declined to the $1-$ to $5-\mathrm{mSv}$ range, depending on whether the study is prospectively or retrospectively gated (60); diagnostic ICA delivers a mean effective radiation dose of approximately 2-2.5 mSv (61). Dose reduction techniques continue to be improved, further reducing radiation exposure, but the risks of higher radiation doses must be considered in decisions about the appropriate testing modality for a particular patient.

\section{CONCLUSION}

CTA has great utility in the domain of clinical heart failure. For diagnosing heart failure, differentiating between etiologies, and planning and monitoring various treatment modalities, CTA provides a quick, noninvasive, and comprehensive analysis of native cardiac and implanted device structure and function as well as a detailed evaluation of both arterial and venous vasculature. Moreover, with the ongoing development and advancement of noninvasive flow calculations, CTA may be able to provide a hemodynamic assessment of heart failure pathophysiology in the future.

\section{DISCLOSURE}

Harvey S. Hecht is a Philips Medical Systems Consultant. No other potential conflict of interest relevant to this article was reported.

\section{REFERENCES}

1. Yancy CW, Jessup M, Bozkurt B, et al. 2013 ACCF/AHA guideline for the management of heart failure: a report of the American College of Cardiology Foundation/American Heart Association Task Force on practice guidelines. Circulation. 2013;128:e240-e327.

2. Go AS, Mozaffarian D, Roger VL, et al. Heart disease and stroke statistics: 2014 update-a report from the American Heart Association. Circulation. 2014;129: e28-e292.

3. Levy D, Kenchaiah S, Larson MG, et al. Long-term trends in the incidence of and survival with heart failure. N Engl J Med. 2002;347:1397-1402.

4. Henneman MM, Schuijf JD, Jukema JW, et al. Assessment of global and regional left ventricular function and volumes with 64-slice MSCT: a comparison with 2D echocardiography. J Nucl Cardiol. 2006;13:480-487.

5. Raman SV, Shah M, McCarthy B, Garcia A, Ferketich AK. Multi-detector row cardiac computed tomography accurately quantifies right and left ventricular size and function compared with cardiac magnetic resonance. Am Heart J. 2006; 151:736-744.

6. Asferg C, Usinger L, Kristensen TS, Abdulla J. Accuracy of multi-slice computed tomography for measurement of left ventricular ejection fraction compared with cardiac magnetic resonance imaging and two-dimensional transthoracic echocardiography: a systematic review and meta-analysis. Eur J Radiol. 2012; 81:e757-e762.

7. Sharma A, Einstein AJ, Vallakati A, et al. Meta-analysis of global left ventricular function comparing multidetector computed tomography with cardiac magnetic resonance imaging. Am J Cardiol. 2014;113:731-738.

8. Feiring AJ, Rumberger JA, Reiter SJ, et al. Sectional and segmental variability of left ventricular function: experimental and clinical studies using ultrafast computed tomography. J Am Coll Cardiol. 1988;12:415-425.

9. MacMillan RM, Rees MR, Weiner R, Maranhao V. Assessment of global and regional left ventricular function in ischemic heart disease using ultrafast computed tomography. Cathet Cardiovasc Diagn. 1988;14:248-254.

10. Nakazato R, Tamarappoo BK, Smith TW, et al. Assessment of left ventricular regional wall motion and ejection fraction with low-radiation dose helical dualsource CT: comparison to two-dimensional echocardiography. J Cardiovasc Comput Tomogr. 2011;5:149-157.

11. Lessick J, Mutlak D, Rispler S, et al. Comparison of multidetector computed tomography versus echocardiography for assessing regional left ventricular function. Am J Cardiol. 2005;96:1011-1015.

12. Yamamuro M, Tadamura E, Kubo S, et al. Cardiac functional analysis with multi-detector row $\mathrm{CT}$ and segmental reconstruction algorithm: comparison with echocardiography, SPECT, and MR imaging. Radiology. 2005;234:381-390.

13. Plumhans C, Muhlenbruch G, Rapaee A, et al. Assessment of global right ventricular function on 64-MDCT compared with MRI. AJR. 2008;190:1358-1361.

14. Taylor AJ, Cerqueira M, Hodgson JM, et al. ACCF/SCCT/ACR/AHA/ASE/ ASNC/NASCI/SCAI/SCMR 2010 Appropriate Use Criteria for Cardiac Computed Tomography: a report of the American College of Cardiology Foundation Appropriate Use Criteria Task Force, the Society of Cardiovascular Computed Tomography, the American College of Radiology, the American Heart Association, the American Society of Echocardiography, the American Society of Nuclear Cardiology, the North American Society for Cardiovascular Imaging, the Society for Cardiovascular Angiography and Interventions, and the Society for Cardiovascular Magnetic Resonance. Circulation. 2010;122:e525-e555.

15. Boogers MJ, van Werkhoven JM, Schuijf JD, et al. Feasibility of diastolic function assessment with cardiac CT: feasibility study in comparison with tissue Doppler imaging. JACC Cardiovasc Imaging. 2011;4:246-256.

16. Lloyd-Jones DM, Larson MG, Leip EP, et al. Lifetime risk for developing congestive heart failure: the Framingham Heart Study. Circulation. 2002;106:30683072 .

17. Allman KC, Shaw LJ, Hachamovitch R, Udelson JE. Myocardial viability testing and impact of revascularization on prognosis in patients with coronary artery disease and left ventricular dysfunction: a meta-analysis. J Am Coll Cardiol. 2002;39:1151-1158.

18. Wallis DE, O'Connell JB, Henkin RE, Costanzo-Nordin MR, Scanlon PJ. Segmental wall motion abnormalities in dilated cardiomyopathy: a common finding and good prognostic sign. J Am Coll Cardiol. 1984;4:674-679.

19. Jolly SS, Amlani S, Hamon M, Yusuf S, Mehta SR. Radial versus femoral access for coronary angiography or intervention and the impact on major bleeding and ischemic events: a systematic review and meta-analysis of randomized trials. Am Heart J. 2009;157:132-140.

20. Abunassar JG, Yam Y, Chen L, D'Mello N, Chow BJ. Usefulness of the Agatston score $=0$ to exclude ischemic cardiomyopathy in patients with heart failure. $\mathrm{Am}$ J Cardiol. 2011;107:428-432. 
21. Budoff MJ, Shavelle DM, Lamont DH, et al. Usefulness of electron beam computed tomography scanning for distinguishing ischemic from nonischemic cardiomyopathy. J Am Coll Cardiol. 1998;32:1173-1178.

22. Sousa PA, Bettencourt N, Dias Ferreira N, et al. Role of cardiac multidetector computed tomography in the exclusion of ischemic etiology in heart failure patients. Rev Port Cardiol. 2014;33:629-636.

23. Raff GL, Gallagher MJ, O'Neill WW, Goldstein JA. Diagnostic accuracy of noninvasive coronary angiography using 64-slice spiral computed tomography. J Am Coll Cardiol. 2005;46:552-557.

24. Andreini D, Pontone G, Pepi M, et al. Diagnostic accuracy of multidetector computed tomography coronary angiography in patients with dilated cardiomyopathy. J Am Coll Cardiol. 2007;49:2044-2050.

25. Bhatti S, Hakeem A, Yousuf MA, Al-Khalidi HR, Mazur W, Shizukuda Y. Diagnostic performance of computed tomography angiography for differentiating ischemic vs nonischemic cardiomyopathy. J Nucl Cardiol. 2011;18:407-420.

26. Chow BJ, Green RE, Coyle D, et al. Computed tomographic coronary angiography for patients with heart failure (CTA-HF): a randomized controlled trial (IMAGE HF Project 1-C). Trials. 2013;14:443.

27. Maisel WH, Stevenson LW. Atrial fibrillation in heart failure: epidemiology, pathophysiology, and rationale for therapy. Am J Cardiol. 2003;91:2D-8D.

28. Vorre MM, Abdulla J. Diagnostic accuracy and radiation dose of CT coronary angiography in atrial fibrillation: systematic review and meta-analysis. Radiology. 2013;267:376-386.

29. Henneman MM, Schuijf JD, Jukema JW, et al. Comprehensive cardiac assessment with multislice computed tomography: evaluation of left ventricular function and perfusion in addition to coronary anatomy in patients with previous myocardial infarction. Heart. 2006;92:1779-1783.

30. Rochitte CE, George RT, Chen MY, et al. Computed tomography angiography and perfusion to assess coronary artery stenosis causing perfusion defects by single photon emission computed tomography: the CORE320 study. Eur Heart J. 2014;35:1120-1130.

31. Williams TJ, Manghat NE, McKay-Ferguson A, Ring NJ, Morgan-Hughes GJ, Roobottom CA. Cardiomyopathy: appearances on ECG-gated 64-detector row computed tomography. Clin Radiol. 2008;63:464-474.

32. Manghat NE, Morgan-Hughes GJ, Marshall AJ, Roobottom CA. Multidetector row computed tomography: imaging congenital coronary artery anomalies in adults. Heart. 2005;91:1515-1522.

33. Bean MJ, Pannu H, Fishman EK. Three-dimensional computed tomographic imaging of complex congenital cardiovascular abnormalities. J Comput Assist Tomogr. 2005;29:721-724.

34. Zeb I, Hamirani YS, Mao S, et al. Detection of aortic regurgitation with 64-slice multidetector computed tomography (MDCT). Acad Radiol. 2010;17:1006-1011.

35. Shah RG, Novaro GM, Blandon RJ, Whiteman MS, Asher CR, Kirsch J. Aortic valve area: meta-analysis of diagnostic performance of multi-detector computed tomography for aortic valve area measurements as compared to transthoracic echocardiography. Int J Cardiovasc Imaging. 2009;25:601-609.

36. Lembcke A, Durmus T, Westermann Y, et al. Assessment of mitral valve stenosis by helical MDCT: comparison with transthoracic Doppler echocardiography and cardiac catheterization. AJR. 2011;197:614-622.

37. Abraham WT, Fisher WG, Smith AL, et al. Cardiac resynchronization in chronic heart failure. N Engl J Med. 2002;346:1845-1853.

38. Cazeau S, Leclercq C, Lavergne T, et al. Effects of multisite biventricular pacing in patients with heart failure and intraventricular conduction delay. $N$ Engl J Med. 2001;344:873-880.

39. Auricchio A, Stellbrink C, Sack S, et al. Long-term clinical effect of hemodynamically optimized cardiac resynchronization therapy in patients with heart failure and ventricular conduction delay. J Am Coll Cardiol. 2002;39:2026-2033.

40. Yu CM, Bax JJ, Monaghan M, Nihoyannopoulos P. Echocardiographic evaluation of cardiac dyssynchrony for predicting a favourable response to cardiac resynchronisation therapy. Heart. 2004;90(suppl 6):vi17-vi22.

41. Buss SJ, Schulz F, Wolf D, et al. Quantitative analysis of left ventricular dyssynchrony using cardiac computed tomography versus three-dimensional echocardiography. Eur Radiol. 2012;22:1303-1309.

42. Van de Veire NR, Marsan NA, Schuijf JD, et al. Noninvasive imaging of cardiac venous anatomy with 64-slice multi-slice computed tomography and noninvasive assessment of left ventricular dyssynchrony by 3-dimensional tissue synchronization imaging in patients with heart failure scheduled for cardiac resynchronization therapy. Am J Cardiol. 2008;101:1023-1029.

43. Singh JP, Houser S, Heist EK, Ruskin JN. The coronary venous anatomy: a segmental approach to aid cardiac resynchronization therapy. J Am Coll Cardiol. 2005;46:68-74.

44. Jongbloed MR, Lamb HJ, Bax JJ, et al. Noninvasive visualization of the cardiac venous system using multislice computed tomography. J Am Coll Cardiol. 2005;45:749-753.

45. Van de Veire NR, Schuijf JD, De Sutter J, et al. Non-invasive visualization of the cardiac venous system in coronary artery disease patients using 64-slice computed tomography. J Am Coll Cardiol. 2006;48:1832-1838.

46. Wong JA, Yee R, Stirrat J, et al. Influence of pacing site characteristics on response to cardiac resynchronization therapy. Circ Cardiovasc Imaging. 2013;6: $542-550$.

47. Moazami N, Hoercher KJ, Fukamachi K, et al. Mechanical circulatory support for heart failure: past, present and a look at the future. Expert Rev Med Devices. 2013;10:55-71.

48. Kirklin JK, Naftel DC, Pagani FD, et al. Sixth INTERMACS annual report: a 10,000-patient database. J Heart Lung Transplant. 2014;33:555-564.

49. Raman SV, Sahu A, Merchant AZ, Louis LB IV, Firstenberg MS, Sun B. Noninvasive assessment of left ventricular assist devices with cardiovascular computed tomography and impact on management. J Heart Lung Transplant. 2010;29:79-85.

50. Acharya D, Singh S, Tallaj JA, et al. Use of gated cardiac computed tomography angiography in the assessment of left ventricular assist device dysfunction. ASAIO J. 2011;57:32-37.

51. Dang NC, Topkara VK, Mercando M, et al. Right heart failure after left ventricular assist device implantation in patients with chronic congestive heart failure. J Heart Lung Transplant. 2006;25:1-6.

52. Garcia-Alvarez A, Fernandez-Friera L, Lau JF, et al. Evaluation of right ventricular function and post-operative findings using cardiac computed tomography in patients with left ventricular assist devices. J Heart Lung Transplant. 2011; 30:896-903.

53. Raman SV, Tran T, Simonetti OP, Sun B. Dynamic computed tomography to determine cardiac output in patients with left ventricular assist devices. J Thorac Cardiovasc Surg. 2009;137:1213-1217.

54. Roger VL, Go AS, Lloyd-Jones DM, et al. Heart disease and stroke statistics: 2012 update-a report from the American Heart Association. Circulation. 2012;125:e2-e220.

55. Keogh AM, Valantine HA, Hunt SA, et al. Impact of proximal or midvessel discrete coronary artery stenoses on survival after heart transplantation. J Heart Lung Transplant. 1992;11:892-901.

56. Mehra MR, Crespo-Leiro MG, Dipchand A, et al. International Society for Heart and Lung Transplantation working formulation of a standardized nomenclature for cardiac allograft vasculopathy-2010. J Heart Lung Transplant. 2010;29: $717-727$.

57. Smart FW, Ballantyne CM, Cocanougher B, et al. Insensitivity of noninvasive tests to detect coronary artery vasculopathy after heart transplant. Am J Cardiol. 1991;67:243-247.

58. Rohnean A, Houyel L, Sigal-Cinqualbre A, To NT, Elfassy E, Paul JF. Heart transplant patient outcomes: 5-year mean follow-up by coronary computed tomography angiography. Transplantation. 2011;91:583-588.

59. Wever-Pinzon O, Romero J, Kelesidis I, et al. Coronary computed tomography angiography for the detection of cardiac allograft vasculopathy: a meta-analysis of prospective trials. J Am Coll Cardiol. 2014;63:19922004.

60. Menke J, Unterberg-Buchwald C, Staab W, Sohns JM, Seif Amir Hosseini A, Schwarz A. Head-to-head comparison of prospectively triggered vs retrospectively gated coronary computed tomography angiography: meta-analysis of diagnostic accuracy, image quality, and radiation dose. Am Heart J. 2013;165:154163.

61. Dill T, Deetjen A, Ekinci O, et al. Radiation dose exposure in multislice computed tomography of the coronaries in comparison with conventional coronary angiography. Int J Cardiol. 2008;124:307-311. 